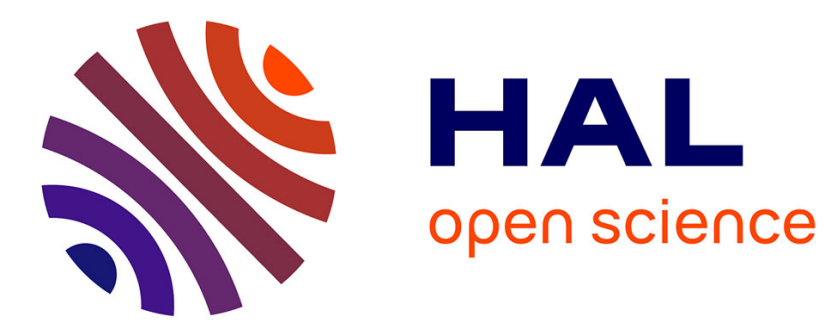

\title{
Vortex dynamics investigation using an acoustic technique
}

S. Manneville, J H Robres, A. Maurel, P. Petitjeans, M. Fink

\section{To cite this version:}

S. Manneville, J H Robres, A. Maurel, P. Petitjeans, M. Fink. Vortex dynamics investigation using an acoustic technique. Physics of Fluids, 1999, 11 (11), pp.3380-3389. 10.1063/1.870197 . hal-02454256

\section{HAL Id: hal-02454256 \\ https://hal.science/hal-02454256}

Submitted on 24 Jan 2020

HAL is a multi-disciplinary open access archive for the deposit and dissemination of scientific research documents, whether they are published or not. The documents may come from teaching and research institutions in France or abroad, or from public or private research centers.
L'archive ouverte pluridisciplinaire HAL, est destinée au dépôt et à la diffusion de documents scientifiques de niveau recherche, publiés ou non, émanant des établissements d'enseignement et de recherche français ou étrangers, des laboratoires publics ou privés. 


\title{
Vortex dynamics investigation using an acoustic technique
}

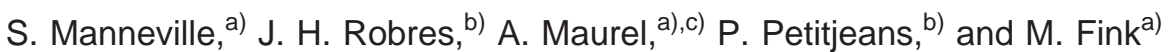 \\ Ecole Supérieure de Physique et de Chimie Industrielles, 10 rue Vauquelin, 75005 Paris, France
}

(Received 5 February 1999; accepted 22 June 1999)

\begin{abstract}
A new acoustic technique using the double time-reversal mirrors system and based on geometrical acoustics, is used to study a vortical flow. The interaction between the sound and a vortex is described in details. This technique has been applied to the study of a stretched vortex. This vortex is generated by stretching the vorticity of a boundary layer in a low velocity water channel. It is shown that the velocity field can be reconstructed from the phase distortion of an ultrasonic wave. The technique gives access to a complete characterization of the vortex dynamics, such as the temporal evolution of its size, its circulation and its position. (C) 1999 American Institute of Physics. [S1070-6631(99)02210-2]
\end{abstract}

\section{INTRODUCTION}

The understanding of vortex dynamics or vorticity distribution in a turbulent flow is of great interest. Indeed, structures with concentrated vorticity, which are observed in various systems from laminar to turbulent regimes, seem to play an important role in the dynamics and in the life of most of turbulent flows. It is now clear that the dynamics of these vortical structures play a role in the intermittence of the energy dissipation of turbulent flows. Intense vortices, which display complex dynamics, have been detected in experimental observations of turbulent flows ${ }^{1}$ as well as in numerical simulations. ${ }^{2-4}$ Most of the time, the vorticity filaments that appear in turbulent regimes are stretched vortices. In that case, resulting vortices are very intense and can concentrate up to $90 \%$ of the whole vorticity field.

Experimentally, these structures and their dynamics are very difficult to investigate. Classical techniques such as hot wire or laser Doppler velocimetry display only local measurements. Stretched vortices are usually nonstationary. They present very small scales (near the dissipation Kolmogorov scale) as well as large scales (integral scale) related to the size of the channel or the size of the "turbulent injector." Strong velocity gradients increase the difficulties to measure the characteristics of such vortices. When the flow has to be seeded for measurements, the particles can migrate and concentrate to particular regions, leaving some other parts almost nonseeded. ${ }^{5}$ In addition, in order to study the dynamics of such vortices, from birth to death, simultaneous, fast, and nonintrusive measurements at different locations and at different times are required. Measurements in the stable and stationary case have been previously performed using laser Doppler velocimetry, leading to a better understanding of the vortex structure in this regime. ${ }^{6}$ To our knowledge, no quantitative measurements have been obtained in the unstable and/or nonstationary case so far.

\footnotetext{
a)Laboratoire Ondes et Acoustique, UMR CNRS 7587.

${ }^{b)}$ Laboratoire de Physique et Mécanique des Milieux Hétérogènes, UMR CNRS 7636.

${ }^{c)}$ Author to whom correspondence should be addressed.
}

In this paper, a new experimental acoustic technique is presented and illustrated by examples of measurements of the characteristics of a stretched vortex.

Indeed, acoustic waves provide a direct, nonintrusive and nonlocalized way of probing hydrodynamic flow fields and several approaches have been proposed in the literature. ${ }^{7-13}$ More recently, Lund et al..$^{14-16}$ have pointed out the possible measurement of vorticity using ultrasound by writing a direct relation between the scattered pressure field and the vorticity field. This relation has been experimentally verified by Baudet $e t$ al. in the case of Benard-Von Kármán flow in air ${ }^{17}$ and has motivated a set of new experiments on wave-flow interaction. ${ }^{18-23}$

The study of sound propagation in turbulent flows is a very active research field both theoretically ${ }^{24-26}$ and experimentally. ${ }^{27-32}$

The acoustic technique presented here is based on geometrical acoustics, an approximation that results from the flow field and the ultrasound characteristics. The timereversal procedure (explained in the paper) allows to amplify the effect of the flow on the wave, leading to very low velocity detection. This technique will be further used to study the structure and the dynamics of this stretched vortex, and this work will be presented in a forthcoming paper. The description as well as the possibilities of such a technique are emphasized here in the complex cases of a stable and an unstable stretched vortex. The first part of this paper describes the hydrodynamic experimental setup. Then, the acoustic system and the way to deduce the velocity field from the acoustical measurements are presented. In the third section, we show an example of our results in the case of a stable, stationary stretched vortex. Finally, the fourth section deals with the more complex case of an unstable, nonstationary vortex.

\section{HYDRODYNAMIC EXPERIMENTAL SETUP}

The experiments are performed in a water channel where the flow is generated by gravity from a constant level tank. The channel itself, of order $2 \mathrm{~m}$ length is made of Plexiglas. It consists of two sections: the first section generates a lami- 


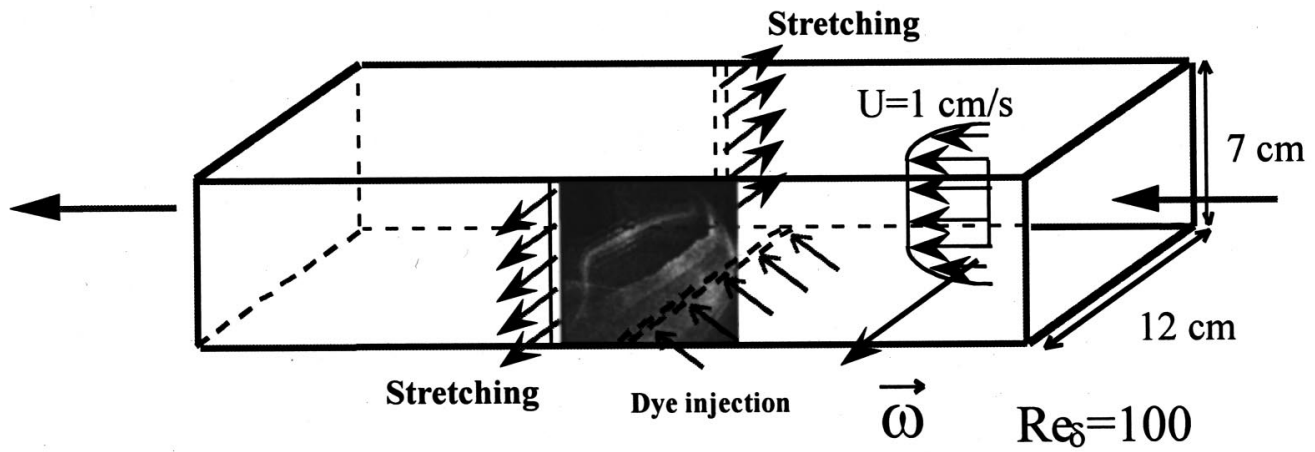

FIG. 1. Experimental setup.

nar flow and the second is the working section. The water enters the first section of the channel through small holes directed perpendicularly to the main flow in order to avoid jet effects. The next section is a $2 \mathrm{D}$ divergent channel of rectangular cross section. It is $60 \mathrm{~cm}$ long and the divergent angle is less than $6^{\circ}$. Next is a channel of $20 \mathrm{~cm}$ long filled with straws aligned in the direction of the flow followed by a 2D convergent section which reduces the cross section to a rectangular section of $12 \mathrm{~cm}$ wide by $7 \mathrm{~cm}$ high.

The purpose of this first part of the channel is to produce a well-controlled laminar flow. The second part of the channel consists of a straight section $60 \mathrm{~cm}$ long (Fig. 1). In the middle of this section, a slot is made in each lateral side and used to create a controlled suction of the main flow. At the end of this section, the flow is evacuated through a system which allows accurate measurement of the flow rate. In the first half of this straight section (before the suction), the laminar flow creates a boundary layer on each side of the channel. Since the suction slots are located on the lateral walls, only the vorticity coming from the boundary layers on the upper and lower walls is stretched by the suction. This stretching is parallel to the initial vorticity. The flow rates of the suctions on each sides are identical, and can be measured and controlled. If the suction is constant at all slots, vortices are generated in phase on the upper wall and on the lower wall, and their vorticity is of opposite sign. ${ }^{33}$ Depending on the suction rate and on the flow rate of the main flow, these vortices interact, merge, or remain separate from each other or from the next pair of vortices generated on the same wall. In the experiments described in this paper, the suction is generated by a hole of $0.6 \mathrm{~cm}$ diameter on each lateral side located $0.5 \mathrm{~cm}$ up from the bottom of the channel (Fig. 2). (a)

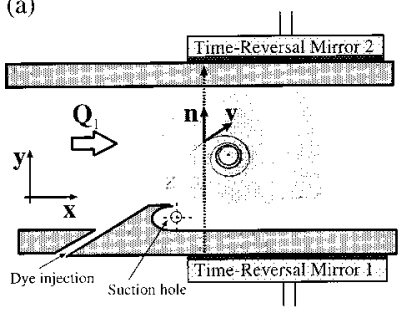

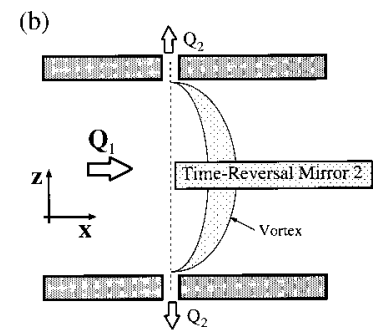

FIG. 2. Schematic view of the positions of the time-reversal mirrors in the channel (a) side view, (b) top view.
The initial vorticity, stretched by these suctions, is enhanced and produces a vortex on the lower wall only. Below a certain flow rate at the exit of the channel, the vortex remains stationary at this location, ${ }^{34}$ sometimes with a low-amplitude precession. Above this flow rate, the vortex is elongated in the direction of the flow and may break up, in which case another one is generated in its place, and so on. In addition, in the experiments reported here a step of $1.1 \mathrm{~cm}$ high shown in Fig. 2 was placed just before the suction. This step produces a more stable vortex with a smaller precessional motion. In the following, $x$ is the axis in the streamwise direction, $y$ is the vertical axis directed from the bottom to the top, and $z$ is the spanwise axis parallel to the initial vorticity. $(0,0,0)$ is the origin at the bottom of the channel, in the stretching axis, and in the center as respect to the spanwise direction. The velocity component are $(U, V, W)$, or $\left(U_{r}, U_{\theta}, U_{z}\right)$ with respect to the center of the vortex. $Q_{1}$ is the downstream flowrate, $Q_{2}$ is the flowrate through each suction slot, the total incoming flow rate being $Q_{1}+2 Q_{2}$.

\section{ACOUSTIC EXPERIMENTAL TECHNIQUE}

Our acoustic technique is based on the interaction between an acoustic wave and the flow in the geometrical acoustics framework. Two 64 transducer arrays of $3.5 \mathrm{MHz}$ central frequency are used. The size of each transducer is $\lambda / 2$ [with $\lambda=0.42 \mathrm{~mm}$ ) in width ( $x$ direction on Fig. 1(a)] and 10 $\mathrm{mm}$ in length ( $z$ direction). Thus, the acoustic field is assumed to be bidimensional in the plane $x-y$ (perpendicular to the large size). Consequently, a plane wave is generated when all transducer of the array are emitting and a cylindrical wave when only one transducer is emitting.

The distance between two consecutive transducers is $0.42 \mathrm{~mm}$. The transducer arrays can receive and emit. The electronic system which pilots the arrays can "time reverse" a wave (see Sec. III B).

\section{A. General context of the sound-flow interaction}

The wavelength $\lambda$ of the ultrasonic wave at $3.5 \mathrm{MHz}$ is $0.42 \mathrm{~mm}$ whereas the characteristic size $d$ of the vortex core is around $5 \mathrm{~mm}$ so that the acoustic ray theory is valid $(\lambda / d \ll 1)$.

Moreover, the characteristic flow velocity is around $U$ $\sim 10 \mathrm{~cm} / \mathrm{s}$. The Mach number is then $M=U / c \sim 10^{-4}$, where $c$ is the speed of sound in the fluid at rest. Under these 
conditions, the flow modifies locally the wave speed, leading to two effects to first order in $M$ : an effect of deflection and an effect of acceleration.

The first effect is due to the vorticity $\boldsymbol{\omega}$ of the flow which locally modifies the direction of the wave propagation, $\mathbf{n}: d \mathbf{n} / d t=\boldsymbol{\omega} \otimes \mathbf{n}$. If $D$ is the distance between the two transducer arrays, the deflection produces a displacement $\delta x$ between the $x$ location of the emitting transducer and the receiving transducer which is of order of magnitude $\delta x$ $\sim M D$. In the experiment described in this paper, the channel height is $7 \mathrm{~cm}$ and the distance $D$ between the transducer arrays is $12 \mathrm{~cm}$, leading to $\delta x \sim 10^{-2} \mathrm{~mm}$. The spatial resolution given by the array pitch being $0.42 \mathrm{~mm}$, the refraction effect is expected to be negligible until about 40 time reversal processes (see Sec. III C). For this reason, a straight-ray propagation is assumed in the following.

The second effect is due to the modification of the local wave speed $\vee$ by the presence of the flow: $\mathbf{v}=c+\mathbf{u} . \mathbf{n}$. The time shift of the acoustic signal after one crossing of the flow is given by $\delta t \sim M L / c$, where $L$ is the characteristic length of the flow. In our experiments, $L$ corresponds to the vortex size $d$. The time shifts are inferred from Fourier transforms of the acoustic signals and the temporal resolution is fixed by the electronic noise level $\delta t \sim 10^{-9} \mathrm{~s}$, which allows a detection of fluid velocities above $10 \mathrm{~cm} / \mathrm{s}$.

It will be seen in the following that the onset of velocity detection is decreased when a time-reversal (TR) procedure is used. Finally, the analysis of the signal (see Sec. IIIC) only includes the effect of acceleration of the ultrasound by the wave in the approximation of geometrical acoustics.

\section{B. The time-reversal process}

The acoustic technique is based on the interaction between the sent ultrasound and the flow. However, because the effects of the flow on the ultrasonic wave are not strong enough, a time-reversal procedure is employed to artificially increase the velocity of the flow (see Sec. III C) so that the effect of the flow becomes measurable. The analysis then consists in the reconstruction of the velocity field from the acoustic signal (see Sec. IV B).

Time-reversal invariance results from the invariance of the wave equation when changing the sign of the time. This invariance has been successfully verified in various media with scalar inhomogeneities, such as temperature or density gradients. ${ }^{35}$ A time-reversal procedure consists in sending a wave, for instance, a plane wave, through a medium by a transducer array, and to record the signal received by the second transducer array (see Fig 3). Then, "time reversing", the wave consists, for each transducer of the second array, in re-emitting the recorded signal after the sign of time has been changed, or, in other words, in emitting first the last part of the received signal. Since the medium is invariant under time reversal, the reversed wave lives again its anterior life until it recovers its initial shape of a plane wave, similarly to a movie seen backwards [Fig. 3(a)].

Time-reversal invariance is broken as soon as the sound speed inhomogeneity depends on the direction of propagation $\mathbf{n}$, i.e., the inhomogeneity is vectorial rather scalar. This (a)
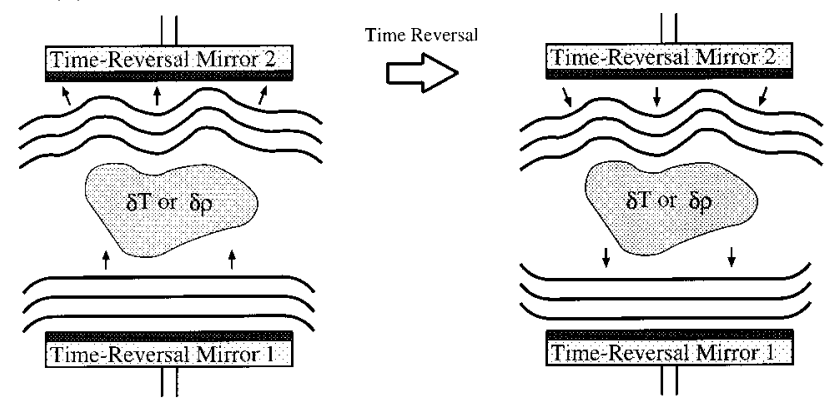

(b)
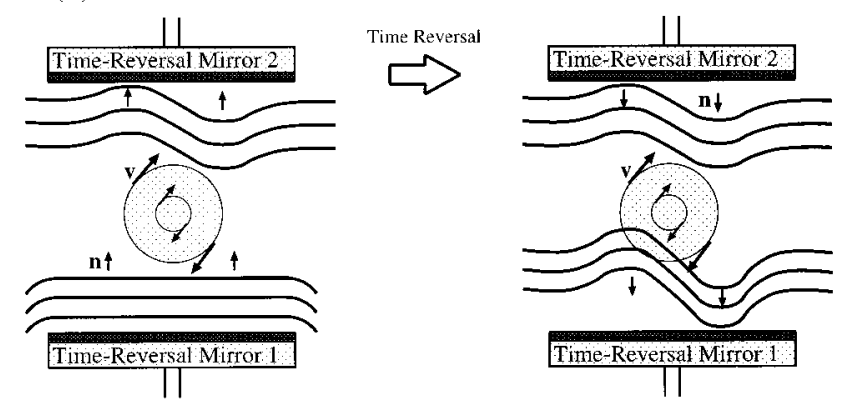

FIG. 3. Time-reversal experiment (a) for a medium with a scalar inhomogeneity (time-reversal invariance) and (b) in the presence of a vortex (breaking of time reversal invariance).

is the case for the velocity of the flow. In such a case, the reversed wave does not recover its initial shape. Instead, the wave front deformation is amplified. In Fig. 3(b), this behavior is illustrated in the case of a vortex insonified by an initially plane wave. Heuristically, the acoustic rays forming the plane wave that cross the left part of the vortex are accelerated, they are the first to reach the receiving array. On the other side, the rays that cross the right side of the vortex are slowed down, they are the last to reach the array. In the TR procedure, the last received part of the signal, on the right side, is sent back first, and will cross a velocity field that, in this direction, speeds it up. Similarly, the first received part, on the left side, is the last to be sent back and will cross a velocity field that slows it down. Consequently, the distortion of the plane wave is increased by the TR procedure. $^{36}$

Note that the whole system flow wave is time reversible. This has been experimentally checked by Roux..$^{21}$ In the experiments described in this paper, the violation of the time reversal invariance is obtained because the time is reversed for the wave and not for the flow.

\section{Using time-reversal mirrors}

We used the so-called double time-reversal mirrors (double TRM) that has been described above (two transducers' arrays located in front of each other on either side of the vortex as shown in Fig. 3). The TR procedure is repeated several times in order to significantly increase the effect of the flow on the wave.

The TRMs, arbitrarily numbered $\mathrm{TRM}_{1}$ and $\mathrm{TRM}_{2}[$ Fig. 3(b)], send simultaneously a plane wave in opposite direc- 
tions. $S_{12}(x)$ is the signal received by $\mathrm{TRM}_{2}$ when $\mathrm{TRM}_{1}$ is the emitter and $S_{21}(x)$ is the signal received by $\mathrm{TRM}_{1}$ when $\mathrm{TRM}_{2}$ is the emitter ( $x$ indicates the transducer position on the arrays). These acoustic signals are then time reversed and simultaneously sent back by the two TRMs, and so on.

A phase shift $\Delta \phi(x)$ is built as follows:

A first "blank" experiment is performed in order to measure the times-of-flight $t_{12}^{0}$ and $t_{21}^{0}$ in absence of flow. Indeed, in a fluid at rest, the acoustic wave is supposed to remain plane after several trips between the TRMs. However, due to the finite aperture of the transducer arrays, the wave fronts are distorted by diffraction effects. ${ }^{36}$ Those reference times-of-flight $t_{12}^{0}$ and $t_{21}^{0}$ are then subtracted to $t_{12}$ and $t_{21}$ measured in the presence of a vortex, yielding the time-shifts $\delta t_{12}$ and $\delta t_{21}$.

The time-shifts $\delta t_{12}$ and $\delta t_{21}$ not only account for the effect of the fluid motion but also for possible effects of the temperature or density inhomogeneities which modify the local speed of sound. However, these last effects do not depend on the direction of the acoustic propagation and cancel out in the difference $\delta t_{12}-\delta t_{21} \cdot{ }^{12,13}$ Note here that $\delta t_{12}$ $+\delta t_{21}$ allows to measure the temperature or concentration gradients without being perturbed by the flow velocity.

The phase shift is defined as: $\Delta \phi(x)=\omega\left[\delta t_{21}(x)\right.$ $\left.-\delta t_{12}(x)\right] / 2$. The dependence of $\Delta \phi(x)$ on the velocity field $\mathbf{u}(\mathbf{r})$ is given by

$$
\begin{aligned}
\Delta \phi(x)= & \frac{\omega}{2}\left(\int_{\mathrm{TRM}_{1}}^{\mathrm{TRM}_{2}} \frac{d y}{(c+\mathbf{u} \cdot \mathbf{n})}-\int_{\mathrm{TRM}_{1}}^{\mathrm{TRM}_{2}} \frac{d y}{c}\right) \\
& -\frac{\omega}{2}\left(\int_{\mathrm{TRM}_{2}}^{\mathrm{TRM}_{1}} \frac{d y}{(c+\mathbf{u} \cdot \mathbf{n})}-\int_{\mathrm{TRM}_{2}}^{\mathrm{TRM}_{1}} \frac{d y}{c}\right)
\end{aligned}
$$

which, at the first order in $M$, reduces to

$$
\Delta \phi(x) \simeq \frac{\omega}{c^{2}} \int_{\mathrm{TRM}_{1}}^{\mathrm{TRM}_{2}} \mathbf{u} \cdot \mathbf{n} d y .
$$

It has been shown in previous works ${ }^{23}$ that the phase shift $\Delta \phi_{N}(x)$ increases linearly with the number $N$ of iterations whereas noise only increases as the square root of $N$. Consequently, the signal-to-noise ratio is enhanced by time reversal. A typical phase shift $\Delta \phi_{N}(x) / N$ is shown in Fig. 5(a) for $N=5$ time-reversal processes.

\section{VORTEX CHARACTERIZATION}

In the experiments described in this section, the flowrate of the suction $Q_{2}$ is fixed, equal to $6.2 \mathrm{l} / \mathrm{min}$ and the downstream flow rate $Q_{1}$ is the control parameter. For small values of $Q_{1}$, the vortex remains almost stationary, located near the small step. Above a threshold $Q_{1 c}=8.7 \mathrm{l} / \mathrm{min}$, the vortex goes unstable: it is advected with the main flow until a critical distance from the step where it breaks down. This behavior leads to periodic cycles of birth (vortex formation near the step), advection and breakdown. In this section, examples of results obtained with the double time-reversal mirrors are presented when the flow rate $Q_{1}$ is below the threshold $Q_{1 c}$. The next section deals with results in the unstable regime of cycles.
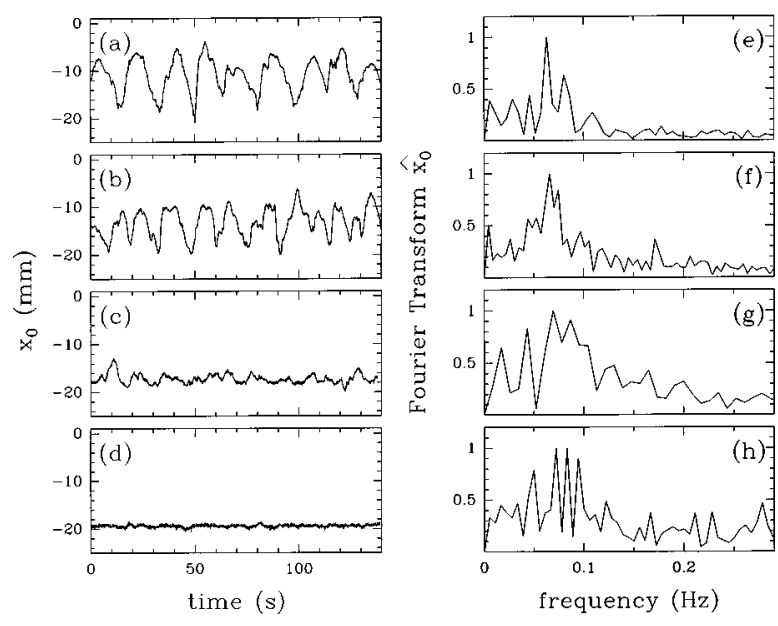

FIG. 4. Study of the precession motion: time series of the $x_{0}$ position (center of the vortex) for $Q_{2}=6.2 \mathrm{\ell} / \mathrm{min}$ and (a) $Q_{1}=8.4 / / \mathrm{min}$, (b) $Q_{1}$ $=7.2 \mathrm{l} / \mathrm{min}$, (c) $Q_{1}=5.1 \mathrm{l} / \mathrm{min}$, (d) $Q_{1}=3.0 \mathrm{l} / \mathrm{min}$ (the position of the suction hole is $x=-35 \mathrm{~mm}$ ). Graphs (e), (f), (g), and (h) are the corresponding normalized Fourier transforms.

\section{A. Study of the precession motion}

For $Q_{1}<Q_{1 c}$, the vortex remains near the step. However, a small precession motion can be observed, which amplitude increases when the flowrate $Q_{1}$ is increased. The double TRMs allows to follow easily this motion. Indeed, the zero value in the phase shift $\Delta \phi\left(x_{0}\right)=0$ corresponds to a ray which crosses the center $x_{0}$ of the vortex. Figure 4(a) shows the temporal variation of $x_{0}$ for different flowrates $Q_{1}$. It can be seen that the precession amplitude increases with $Q_{1}$. Figures 4(b) shows the corresponding Fourier transforms. The frequency is of order $0.1 \mathrm{~Hz}$ and does not seem to depend significantly on the flowrate.

Experiments are in progress in order to characterize the precession as a function of the stretching. In the following, only vortices with a very slow and low amplitude precession will be presented for the velocity reconstruction.

\section{B. Velocity field reconstruction}

As already mentioned, the vortex can be considered as stationary for flowrates small enough. In this case, a reconstruction method to access the velocity field from the phase shift has been developed. An example of phase distortion is shown on Fig. 6(a) for $Q_{1}=1.3 \mathrm{l} / \mathrm{min}$; this signal is obtained from an average on 200 samples on the same vortex. Let us recall that the effect of the velocity is integrated along the acoustic rays on the $y$ direction (sound propagation direction), so that it is not possible to get the $y$ dependence of the velocity $u(y)$. On the $x$ direction, on the contrary, $u(x)$ is obtained with a spatial resolution given by the array pitch $(0.42 \mathrm{~mm})$. In the case of an axisymmetric vortex, this restriction has no consequence thanks to the invariance between $x$ and $y$. This case has been investigated in a previous study. ${ }^{23}$

The axisymmetric reconstruction algorithm consists in dividing the propagation area into $p$ small concentric rings $r \in\left[r_{i+1}, r_{i}\right]$ of size corresponding to the distance between 


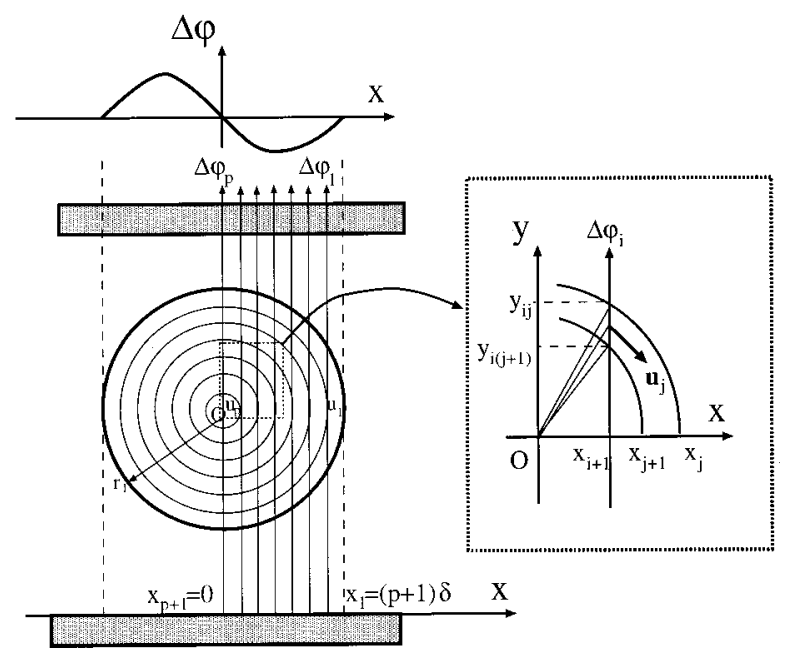

FIG. 5. Reconstruction principle for a circular vortex shape: in each ring, the velocity is taken constant and extracted from the phase profile [Eqs. (4.1) and (4.2)].

two transducers. The orthoradial velocity $u_{\theta}$ is sampled in each ring and takes the values $u_{i}$ (see Fig. 5).

$r_{1}$ is the edge of the vortex, defined as the first point (coming from outside the vortex) where the phase shift $\Delta \phi(x)$ is not zero. $r_{p+1}$ is the center of the vortex, where the phase shift is also zero [all the velocities $u_{\theta}(r)$ are perpendicular to the ray direction that crosses the vortex center], and $p+2$ is the number of experimental data points. The phase distortion $\Delta \phi_{i}=\Delta \phi\left(x_{i+1}\right)$ are sampled at the transducer position $x_{i+1}$.

In the first ring $r_{1}$, the velocity $u_{1}$ is deduced from the experimental phase shift $\Delta \phi_{1}$ by a relation resulting from Eq. (3.1): $\Delta \phi_{1}=2 \omega / c^{2} r_{2} \ln \left(\left(\sqrt{r_{1}^{2}-r_{2}^{2}}+r_{1}\right) / r_{2}\right) u_{1}$. Then, in the second ring $r_{2}$, the velocity $u_{2}$ can be obtained from $\Delta \phi_{2}$ and the velocity $u_{1}$ previously calculated at the previous step: $\Delta \phi_{2}=2 \omega / c^{2}\left\{r_{2} \ln \left[\left(\sqrt{r_{1}^{2}-r_{3}^{2}}+r_{1}\right) /\left(\sqrt{r_{2}^{2}-r_{3}^{2}}\right.\right.\right.$ $\left.\left.\left.+r_{2}\right)\right] u_{1}+r_{3} \ln \left[\left(\sqrt{r_{2}^{2}-r_{3}^{2}}+r_{2}\right) / r_{3}\right] u_{2}\right\}$. This process is repeated until the center of the vortex is reached using the general relation:

$$
\Delta \phi_{i}=\mathcal{M}_{i j} u_{j},
$$

where

$$
\begin{aligned}
& \mathcal{M}_{i j}=\frac{2 \omega}{c^{2}} r_{j+1} \ln \frac{\sqrt{r_{j}^{2}-r_{i+1}^{2}}+r_{j}}{\sqrt{r_{j+1}^{2}-r_{i+1}^{2}}+r_{j+1}} \text { if } i \geqslant j, \\
& \mathcal{M}_{i j}=0 \quad \text { if } \quad i<j .
\end{aligned}
$$

$\mathcal{M}$ is an invertible, triangular matrix. Thus, $u$ can be reconstructed from the measurements of $\Delta \phi$.

This assumes that the vortex is axisymmetric, which is not exactly the case in our experiment. Indeed, the vortex has a slightly elliptical shape. It can be seen in Fig. 2 that, far from the vortex core, the shape is clearly non axisymmetric and, from these external contours, should rather be called elliptical. However, closer to the core, the streamlines become circular. Actually, visualizations only give a qualitative idea of the vortex shape but carry no information on the velocity distribution.

In this case, a possible way to obtain a bidimensional reconstruction is to make an assumption on the vortex shape coming from visualizations for instance. We chose an alternative solution by mixing two axisymmetric reconstructions for both half parts of the phase distortion: $\phi_{r}$ (right) for the negative phase distortion ( $n_{r}$ elements) and $\phi_{l}$ (left) for the positive phase distortion ( $n_{l}$ elements, with $n_{r}+n_{l}=64$ transducers). The velocity profiles $u_{r}$ and $u_{l}$ are independently obtained by inverting the $\mathcal{M}_{l}$ and $\mathcal{M}_{r}$. A typical phase distortion is shown in Fig. 6(a) and the corresponding reconstructed half profiles $u_{r}$ and $u_{l}$ on solid line in Fig. 6(b) for $Q_{1}=1.3 \mathrm{\ell} / \mathrm{min}$. Such a vortex shape is not continuous: it can be seen that the right part is $18 \mathrm{~mm}$ long whereas the left part is $12 \mathrm{~mm}$ long. An elliptical shape is used to correct this discontinuity and to approach the real shape of the vortex (see Fig. 7). The values of the velocity are then corrected with the help of this new shape [curve in dashed line on Fig. 6(b)].
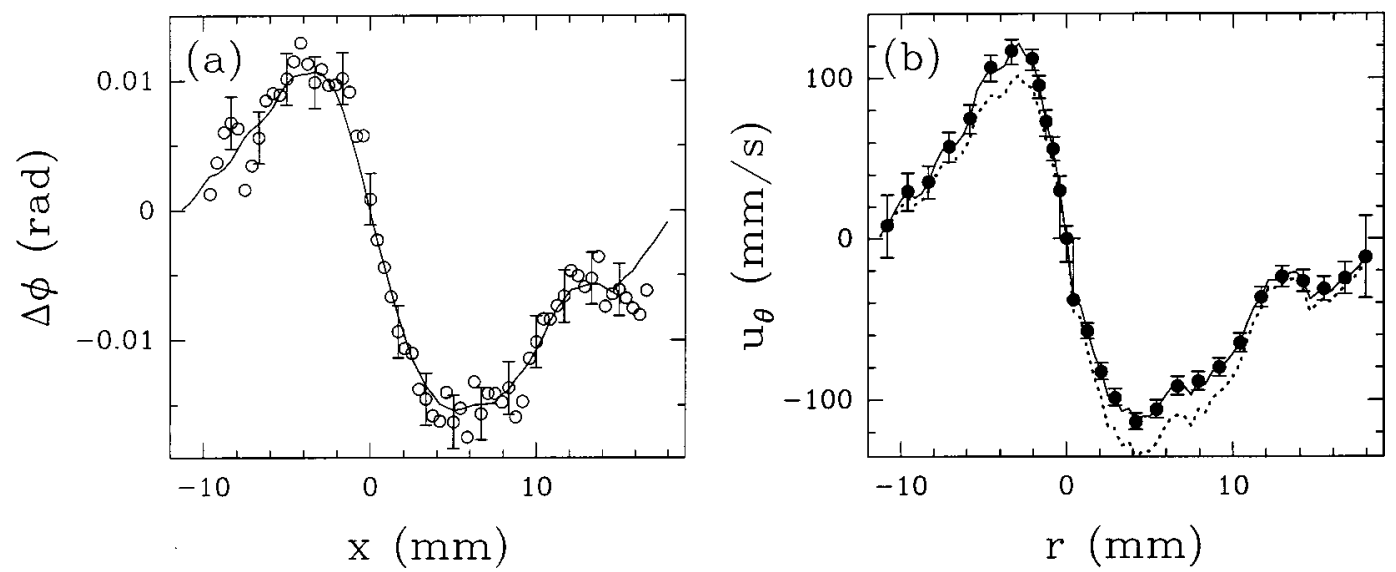

FIG. 6. (a) Phase distortion as a function of the transducer position $x$ for $Q_{1}=1.3 \ell / \mathrm{min}$ and $Q_{2}=6.2 \ell / \mathrm{min}$. (b) Reconstructed orthoradial velocity as a function of the radius $r$. 


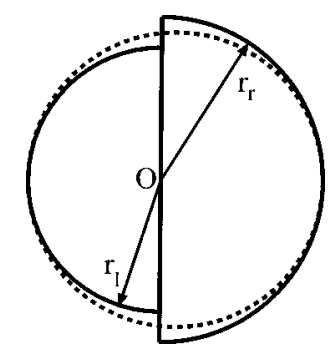

FIG. 7. From the two circular reconstructions to the elliptical shape closer to the real vortex.

\section{ACCESSING THE VORTEX DYNAMICS}

\section{A. Determination of the vortex characteristics}

The relevant characteristics of the vortex are the diameter of the vortex and its circulation, or its diameter and its maximum velocity.

Let $r_{m}$ be the radius where the velocity is maximum and takes the value $U_{m}$, and $x_{m}$ the transducer position where the phase shift is maximum and takes the value $\Delta \phi_{m}$.

$\Delta \phi(x)$ and $U(r)$ can be written:

$$
\begin{aligned}
& \Delta \phi(x)=\Delta \phi_{m} f\left(\tilde{x}, \operatorname{Re}, \operatorname{Re}_{\text {stretching }}\right), \\
& u(r)=U_{m} g\left(\widetilde{r}, \operatorname{Re}, \operatorname{Re}_{\text {stretching }}\right),
\end{aligned}
$$

where $\tilde{x}=x / r_{m}$ and $\tilde{r}=r / r_{m}$ (so that $\tilde{y}=y / r_{m}$ ), $\operatorname{Re}$ is the Reynolds number of the mean flow and $\mathrm{Re}_{\text {stretching }}$ is the Reynolds number based on the stretching velocity and the diameter of the hole through which the stretching is produced.
If the distance between the two transducer arrays is large enough, the phase shift can be written:

$$
\Delta \phi(x)=\frac{\omega}{c^{2}} \int_{-\infty}^{+\infty} \mathbf{u}(r) . \mathbf{n} d y
$$

which becomes

$$
\begin{aligned}
& \Delta \phi_{m} f\left(\widetilde{x}, \operatorname{Re}, \operatorname{Re}_{\text {stretching }}\right) \\
&\left.\quad=r_{m} U_{m} \frac{\omega}{c^{2}} \int_{-\infty}^{+\infty} g\left(\widetilde{r}, \operatorname{Re}, \operatorname{Re}_{\text {stretching }}\right)\right) \frac{\tilde{x}}{\widetilde{r}} d \widetilde{y} .
\end{aligned}
$$

This relation implies that the ratio $\Delta \phi_{m} / r_{m} U_{m}$ depends only on the two Reynolds numbers ( $\operatorname{Re}$ and $\mathrm{Re}_{\text {stretching }}$ ). The circulation $\Gamma=\int_{c} \mathbf{u}(r) \cdot \mathbf{d} r \sim 2 \pi r_{m} U_{m}$ is then obtained only from the measurement of $\Delta \phi_{m}$ by the relation:

$$
\Gamma=\Delta \phi_{m} F\left(\operatorname{Re}, \operatorname{Re}_{\text {stretching }}\right) .
$$

The diameter of the vortex $2 r_{m}$ can be deduced from the measurement of $x_{m}$ using the same relations and the fact that the position $x_{1}$ of the zero phase shift corresponds to the radius $r_{1}$ where the velocity is also zero (see Fig. 5):

$$
r_{m}=x_{m} G\left(\operatorname{Re}, \mathrm{Re}_{\text {stretching }}\right) \text {. }
$$

Thus, it is sufficient for the characterization of the vortex to extract only $x_{m}$ and $\Delta \phi_{m}$ from the phase shift profile. The
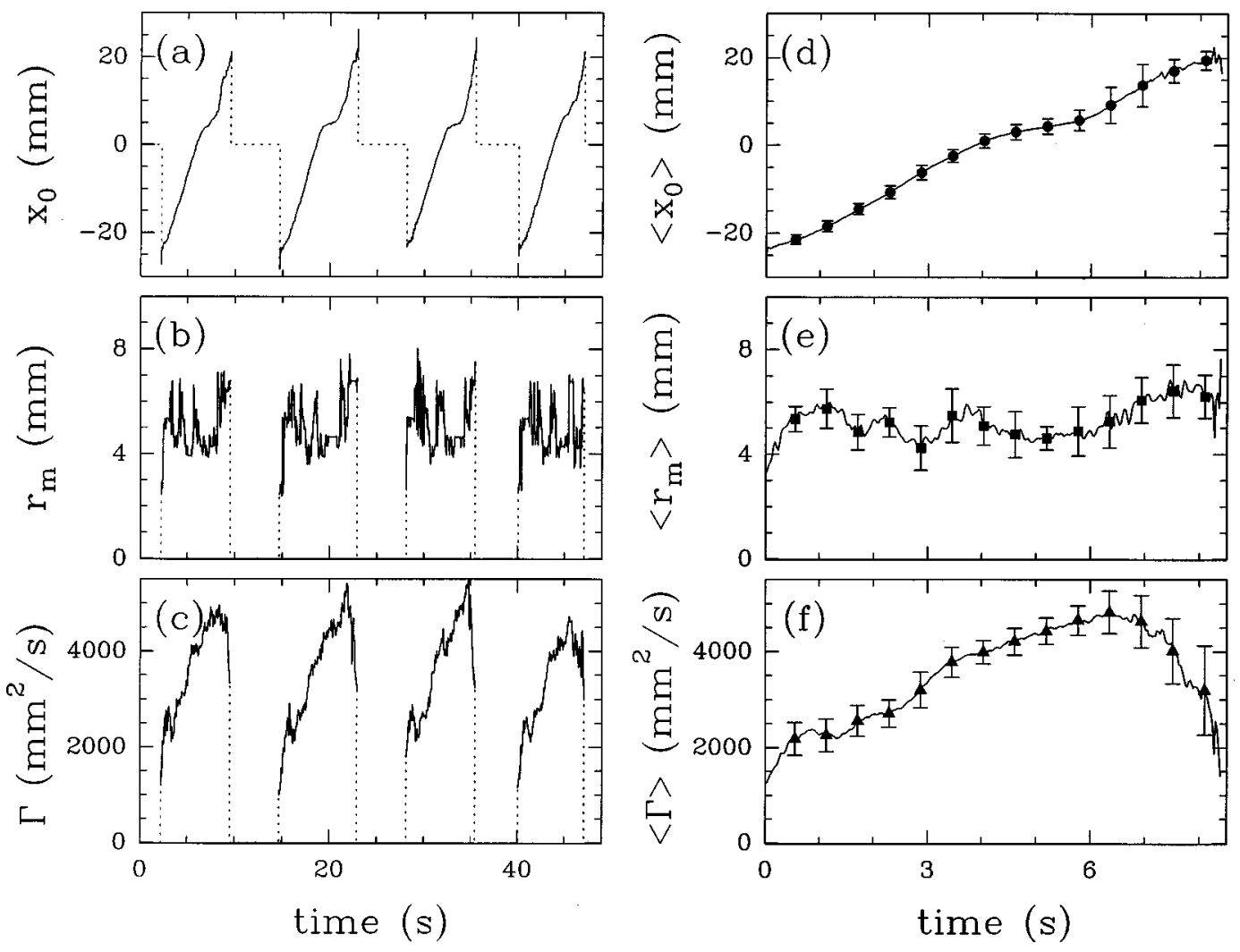

FIG. 8. Temporal evolution of (a) $x_{0}$, location of the center of the vortex, (b) $r_{m}$, the vortex radius, and (c) $\Gamma$, the circulation of the vortex for four consecutive cycles. (d), (e), and (f) show the average evolution of these parameters on 20 cycles. $Q_{1}=14 \ell / \mathrm{min}, Q_{2}=6.2 \ell / \mathrm{min}$. 

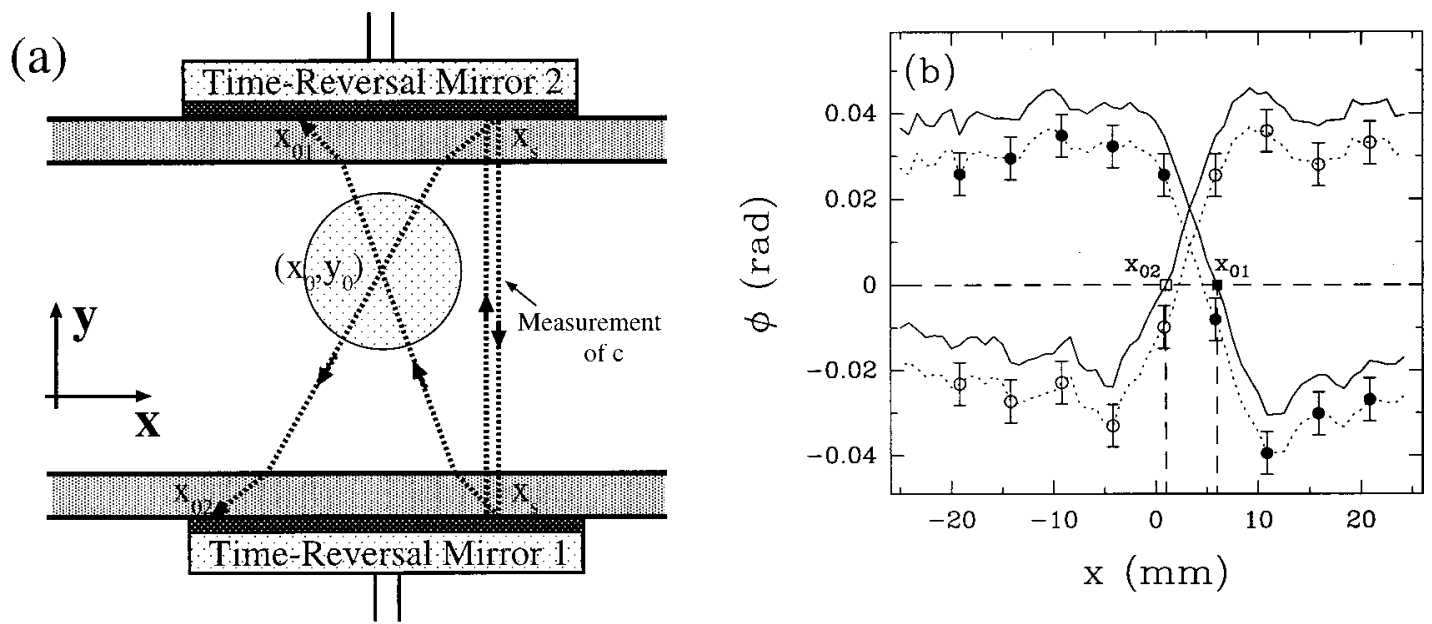

FIG. 9. (a) Schematic view of acoustic ray trajectories in the case of cylindrical waves. The $x_{s}-x_{s}$ rays are used to measure the sound speed. The $x_{s}-x_{01}$ and $x_{s}-x_{02}$ rays are used to locate the center $\left(x_{0}, y_{0}\right)$ of the vortex. (b) The experimental phase distortions $\phi_{12}(x)$ and $\phi_{21}(x)$ are plotted as a function of $x$ in dashed lines. The solid lines represent the phase shift $\phi_{\mathrm{v}, 12}(x)$ and $\phi_{\mathrm{v}, 21}(x)$ related to the vortex only, without the contribution of the propagation term. The $x$ positions $x_{01}$ and $x_{02}$, where $\phi_{\mathrm{v}, 12}$ and $\phi_{\mathrm{v}, 21}$ vanish allow to deduce the position $\left(x_{0}, y_{0}\right)$ of the vortex center. $Q_{1}=12.4 \ell / \mathrm{min}, Q_{2}=6.2 \ell / \mathrm{min}$.

functions $F$ and $G$ have to be calculated only once for each given flowrate and stretching. If the vortex is not symmetric, an average value is taken.

\section{B. Example of vortex dynamics}

The instantaneous phase distortion is recorded as a function of time. $F$ and $G$ are deduced from the first profile. Then, $x_{0}, x_{m}$, and $\Delta \phi_{m}$ are extracted at each step and $r_{m}$ and $\Gamma$ are calculated.

Figure 8 shows the temporal evolution of these characteristics on a few periods of formation, advection and breakdown of a vortex.

In Figs. 8(d), 8(e), and 8(f), these characteristics have been plotted on a mean cycle (averaging over 20 cycles).

It can be seen that the vortex size remains roughly constant while the circulation increases until a maximum value before the vortex breaks down. The vortex is first advected with a constant velocity then slows down a little bit before it breaks.

\section{Bidimensional tracking using cylindrical waves}

The previous study was performed using plane waves. This was done for various reasons: the signal amplitude is higher because all the transducers are used, and the signalto-noise ratio is better because time reversal process can be used.

However, we have seen that the vortex tracking using plane waves is only possible in one direction: the $x$ direction along the chanel. In order to get the trajectory of the vortex in the $x-y$ plane, another process using cylindrical waves has been developed. In that case, the time reversal process cannot be used any longer: the only effect of a time-reversal procedure would be the defocalization of the reversed wave without increasing the signal-to-noise ratio. ${ }^{36}$

Only one transducer on each array emits. The sources are located in front of each other [same position $x_{s}$ on the arrays, see Fig. 9(a)]. The received signals on both arrays are written as

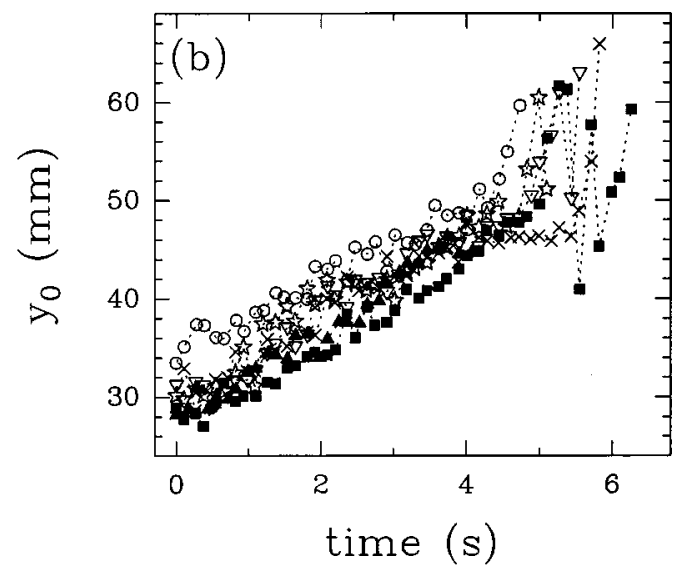

FIG. 10. Temporal evolution of the position (a) $x_{0}$ and (b) $y_{0}$ of the center of the vortex for 6 different cycles where $Q_{1}=12.4 \ell / \mathrm{min}$ and $Q_{2}$ $=6.2 \ell / \mathrm{min}$ 
(a)

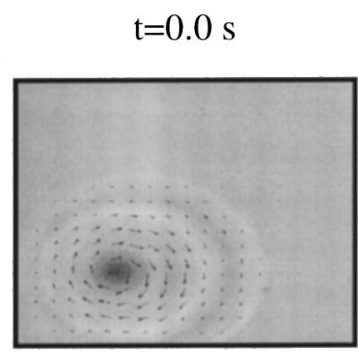

$\mathrm{t}=3.4 \mathrm{~s}$

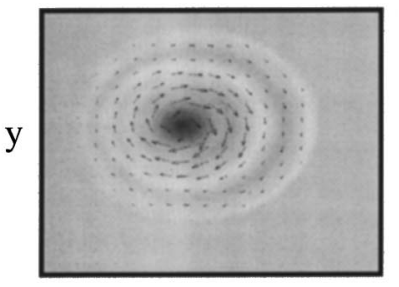

$\mathrm{X}$

(b)
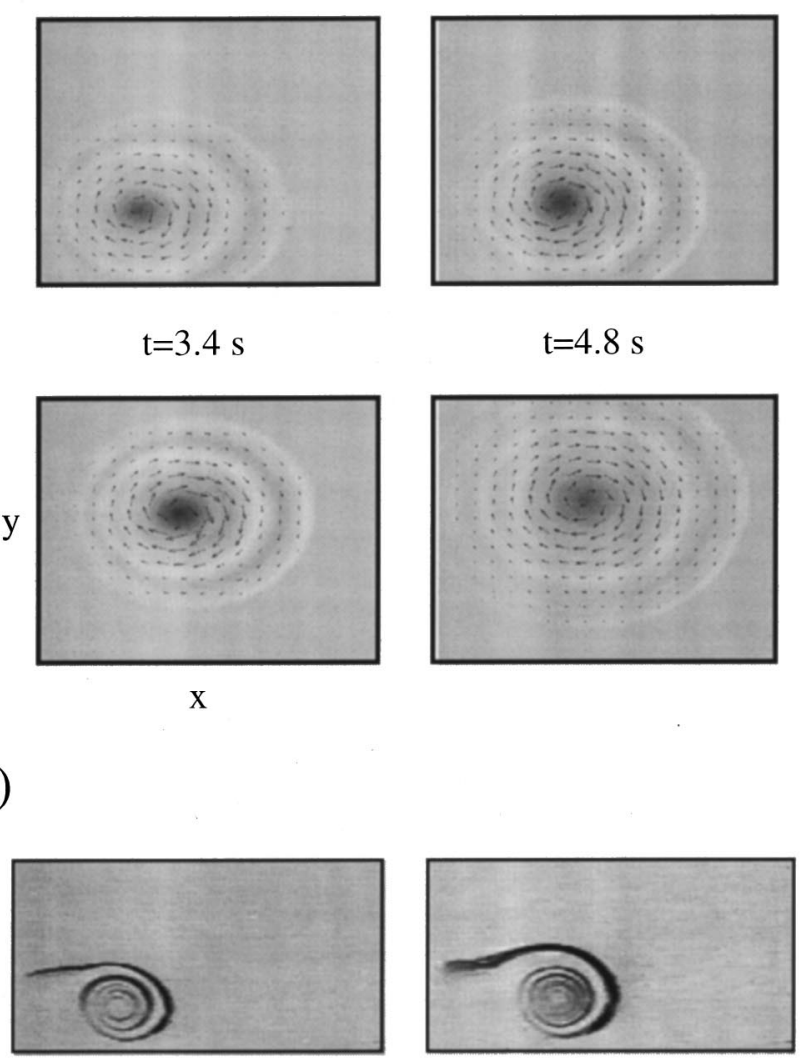

$\mathrm{t}=4.8 \mathrm{~s}$
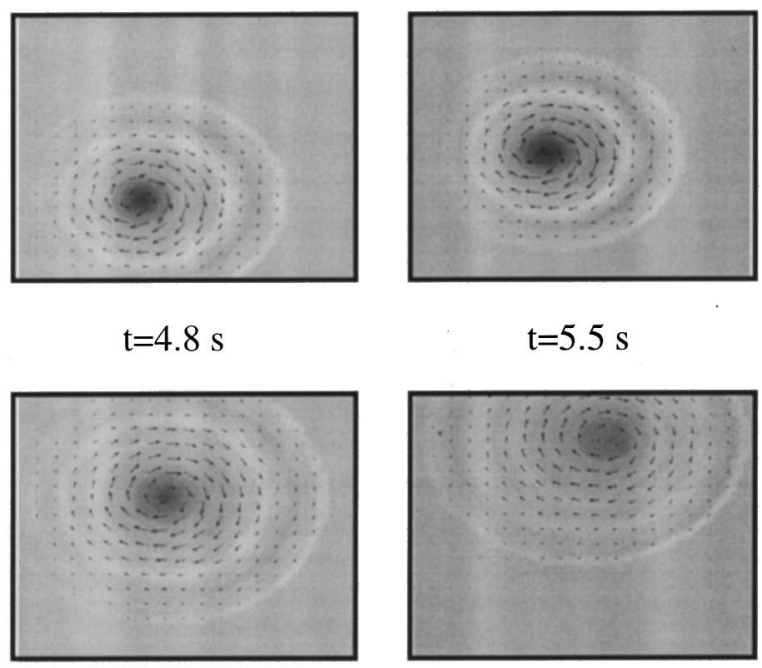

$t=5.5 s$
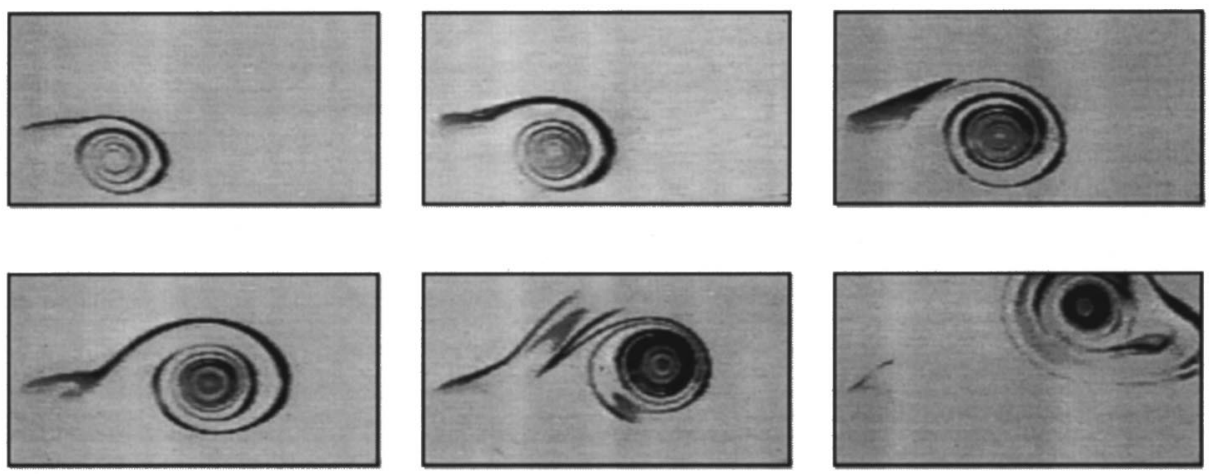

vorticity $\left(\mathrm{s}^{-1}\right)$
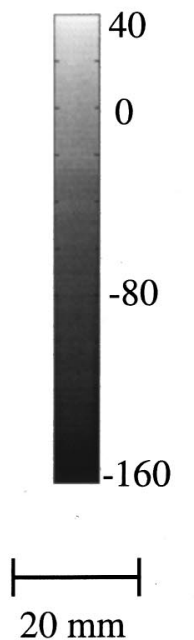

FIG. 11. (a) Sequence of pictures showing the vorticity field (gray level), the velocity field (arrays) and the motion of the vortex. The vorticity and velocity are deduced from the maximum phase shift in the case of plane waves, so that the values are taken with an arbitrary scale. (b) Sequence of experimental pictures: the vortex is visualized with fluorescent dye.

$$
\begin{aligned}
\phi(\theta) & =\frac{\omega L}{c+U_{\text {mean }} \cos \theta}-\frac{\omega}{c^{2}} \int_{0}^{L} \mathbf{u}(r) \cdot \mathbf{n} d l \\
& \sim \frac{\omega L}{c}-\frac{\omega L U_{\text {mean }} \cos \theta}{c^{2}}-\frac{\omega}{c^{2}} \int_{0}^{L} \mathbf{u}(r) \cdot \mathbf{n} d l
\end{aligned}
$$

where $U_{\text {mean }}$ is the mean velocity in the chanel (constructed with $\left.Q_{1}\right), \theta$ is the angle $\left(\mathbf{U}_{\text {mean }}, \mathbf{n}\right)$ and $L$ is the distance between emitter and receiver: $L=D / \sin \theta$ (see Fig. 9).

In the case of plane waves described before, $\theta=\pi / 2$ so that the dependence of $\phi$ with $U_{\text {mean }}$ cancels out. On the other hand, the reverse signal allows to eliminate the first term $\omega L / c$ by subtracting the direct and reverse signals (see Sec. III C).

In the case of cylindrical waves, $c$ has to be determined. For this, we use the direct $\phi_{12}$ and reverse $\phi_{21}$ signals as described in Fig. 9: $c=2 \omega D /\left(\phi_{12}+\phi_{12}\right)$. The second term $\omega L U_{\text {mean }} \cos \theta / c^{2}$ is much smaller that the others in our ex- periment and can be neglected, mainly because $\theta$ remains close to $\pi / 2$ : $\quad \omega L U_{\text {mean }} \cos \theta / c^{2} \sim 10^{-3} \mathrm{rad}$, whereas $\left(\omega / c^{2}\right) \int_{0}^{L} \mathbf{u}(r) \cdot \mathbf{n} d l$ is of order $10^{-2} \mathrm{rad}$.

The experimental phase distortions $\phi_{12}(x)$ and $\phi_{21}(x)$ are plotted in Fig. 9 in dashed lines. The solid lines represent $\phi_{\mathrm{v}}(x)=\phi(x)-\omega L / c \sim \omega / c^{2} \int_{0}^{L} \mathbf{u}(r) . \mathbf{n} d l$ which is the phase shift related to the vortex only.

The transformation between $x$ and $\theta$ includes the effect of refraction on the interface Plexiglas/water. The positions $x_{01}$ and $x_{02}$ where $\phi_{\mathrm{v}, 12}(x)$ and $\phi_{\mathrm{v}, 21}(x)$ vanish define two acoustic rays crossing the vortex center. From $x_{s}, x_{01}$ and $x_{02}$, we first compute the angles $\theta_{1}$ and $\theta_{2}$ between the $y$ axis and the direction of propagation $\mathbf{n}$ corresponding to those two rays by solving for $\theta_{i}$ in

$$
x_{0 i}-x_{s}=D \tan \theta_{i}+2 d \tan \left[\sin ^{-1}\left(\frac{n_{w}}{n_{p}} \sin \theta_{i}\right)\right] \text { for } i=1,2 \text {, }
$$


where $n_{w}$ and $n_{p}$ are, respectively, the acoustic refractive indices of water and Plexiglas (with $n_{w}=2.7$ and $n_{p}=1.5$ ), and $d$ is the Plexiglas width. The coordinates $\left(x_{0}, y_{0}\right)$ of the vortex center are then given by the intersection of the two rays:

$$
\begin{aligned}
& x_{0}=\frac{1}{2}\left(x_{s}+\frac{\tan \theta_{2} x_{01}+\tan \theta_{1} x_{02}}{\tan \theta_{1}+\tan \theta_{2}}\right), \\
& y_{0}=\frac{1}{2}\left(D+\frac{x_{02}-x_{01}}{\tan \theta_{1}+\tan \theta_{2}}\right) .
\end{aligned}
$$

The values are plotted in Fig. 10 as a function of time for six cycles of a same experiment. The evolution of the different cycles are similar even if they do not superimpose. Figure 11 shows the position, the vorticity and the velocity fields at different times along a cycle.

\section{CONCLUSION}

The acoustic technique presented in this paper has been applied for the first time to the study of the vortex structure and dynamics. The measured phase distortion gives directly the circulation. In the case of a vortex, the velocity field can be deduced with a good accuracy. The measurement is global and non intrusive. It allows the access to the whole vortex dynamics.

The results presented here prove that this technique is relevant for the study of vortices and adds to classical techniques such as laser doppler velocimetry, particles image velocimetry, hot wire, etc.

Most of the other nonintrusive experimental techniques need the injection of particles. The measured velocity is then the velocity of the particles which is not always the flow velocity. It has been shown by Wunenburger et al..$^{5}$ that, in the particular case of vortex flows, the measurements can be biased by the particles demixion. In the case of turbulent flows, the use of particules can be more irrevelant because of the complex behavior of the particles in such a flow.

Acoustical measurements based on flow-wave interaction (without particles) are used by Baudet et al. ${ }^{37}$ for the study of turbulent flows. They show that acoustic measurements directly obtained in the Fourier space give access to the spatial scales of the flow (without localization of the structures). Work is in progress to use this time-scale acoustic interferometry technique on vortex breakdown.

\section{ACKNOWLEDGMENTS}

We would like to thank Eduardo Wesfreid and Claire Prada for fruitful discussions.

${ }^{1}$ O. Cadot, S. Douady, and Y. Couder, "Characterization of the lowpressure filaments in a three-dimensional turbulent shear flow," Phys. Fluids 7, 630 (1995).

${ }^{2}$ E. D. Siggia, "Numerical study of small-scale intermittency in threedimensional turbulence," J. Fluid Mech. 107, 375 (1991).
${ }^{3}$ M. E. Brachet, "'Direct simulations of three-dimensional turbulence in the Taylor-Green vortex," Fluid Dyn. Res. 8, 1 (1991).

${ }^{4}$ J. Jimenez, A. A. Wray, P. G. Saffman, and S. Rogallo, “The structure of instense vorticity in isotropic turbulence," J. Fluid Mech. 255, 65 (1993).

${ }^{5}$ R. Wunenburger, B. Andreotti, and P. Petitjeans, "Influence of precession on velocity measurements in a strong laboratory vortex," Exp. Fluids 26 (1999).

${ }^{6}$ P. Petitjeans, J. H. Robres, J. E. Wesfreid, and N. Kevlahan, "Experimental evidence for a new type of stretched vortex," Eur. J. Mech. B/Fluids 17, 549 (1998).

${ }^{7}$ P. R. Gromov, A. B. Ezerskii, and A. L. Fabrikant, "'Sound scattering by a vortex wake behind a cylinder," Sov. Phys. Acoust. 28, 552 (1982).

${ }^{8}$ A. L. Fabrikant, "Sound scattering by vortex flows," Sov. Phys. Acoust. 29, 152 (1983).

${ }^{9}$ P. V. Sakov, "Sound scattering by a vortex filament," Acoust. Phys. 39, 280 (1993).

${ }^{10}$ D. W. Schmidt and P. L. Tilman, "Experimental study of sound wave phase fluctuations caused by turbulent wakes," J. Acoust. Soc. Am. 47, 1310 (1970).

${ }^{11}$ R. H. Engler, D. W. Schmidt, W. Wagner, and B. Weitemeier, "Nondisturbing acoustical measurement of flow fields; new developments and applications," J. Acoust. Soc. Am. 85, 72 (1989).

${ }^{12}$ K. B. Winters and D. Rouseff, "Tomographic reconstruction of stratified fluid flow,' IEEE Trans. Ultrason. Ferroelectr. Freq. Control 40, 26 (1993).

${ }^{13}$ S. A. Johnson, J. F. Greenleaf, M. Tanaka, and G. Flandro, "Reconstructing three-dimensional temperature and fluid velocity vector fields from acoustic transmission measurements,", ISA Trans. 16, 3 (1997).

${ }^{14} \mathrm{~F}$. Lund and C. Rojas, "Ultrasound as a probe of turbulence," Physica D 37, 508 (1989).

${ }^{15} \mathrm{H}$. Contreras and F. Lund, "Ultrasound as a probe of turbulence. II, Temperature inhomogeneities,'” Phys. Lett. B 149, 127 (1990).

${ }^{16} \mathrm{R}$. Berthet and F. Lund, "The forward scattering of sound by vorticity," Phys. Fluids 7, 2522 (1995).

${ }^{17}$ C. Baudet, S. Ciliberto, and J. F. Pinton, "Spectral analysis of the Von Kármán flow using ultrasound scattering," Phys. Rev. Lett. 67, 193 (1991).

${ }^{18}$ J. F. Pinton, C. Laroche, S. Fauve, and C. Baudet, "Ultrasound scattering by buoyancy driven flows," J. Phys. II 3, 767 (1993).

${ }^{19}$ F. Vivanco and F. Melo, "Surface waves scattering by a vertical vortex," Nonlinear Dynamics and Acoustics, ETSEIB, 1998, pp. 114-122.

${ }^{20}$ M. Oljaca, X. Gu, A. Glezer, M. Baffico, and F. Lund, "Ultrasound scattering by a swirling jet," Phys. Fluids 10, 886 (1998).

${ }^{21} \mathrm{P}$. Roux and M. Fink, "Experimental evidence in acoustics of the violation of time-reversal invariance induced by vorticity," Europhys. Lett. 32, 25 (1995).

${ }^{22}$ P. Roux, J. de Rosny, M. Tanter, and M. Fink, "The Aharonov-Bohm effect revisited by an acoustic time-reversal mirror,' Phys. Rev. Lett. 79, 3170 (1997)

${ }^{23}$ S. Manneville, A. Maurel, P. Roux, and M. Fink, "Characterization of a large vortex using acoustic time-reversal mirrors,' Eur. Phys. J. B (unpublished).

${ }^{24}$ M. Baffico, D. Boyer, and F. Lund, "Propagation of acoustic waves through a system of many vortex rings," Phys. Rev. Lett. 80, 2590 (1998).

${ }^{25} \mathrm{D}$. Boyer and F. Lund, "Propagation of acoustics waves in disordered flows composed of many vortices" (unpublished).

${ }^{26}$ V. E. Ostashev, "Acoustics in moving inhomogeneous media," edited by E. \& FN SPON, London, ISBN 0419224300 (1997).

${ }^{27}$ A. Petrossian and J. F. Pinton, "Sound scattering on a turbulent, weakly heated jet,"' J. Phys. II 7, 801 (1997).

${ }^{28}$ R. Labbé and J. F. Pinton, "Propagation of sound through a turbulent vortex," Phys. Rev. Lett. 81, 1413 (1998).

${ }^{29}$ B. Dernoncourt, J. F. Pinton, and S. Fauve, "Experimental study of vorticity filaments in turbulent swirling flows," Physica D 117, 181 (1998).

${ }^{30} \mathrm{~B}$. Lipkens and P. Blanc-Benon, "Propagation of finite amplitude sound through turbulence: a geometric acoustics approach," C. R. Acad. Sci., Ser. IIb: Mec., Phys., Chim., Astron. 320, 477 (1995).

${ }^{31}$ P. Chevret, P. Blanc-Benon, and D. Juvé, “A numerical model for sound propagation through a turbulent atmosphere near the ground," J. Acoust. Soc. Am. 100, 3587 (1996).

${ }^{32}$ V. E. Ostashev, P. Blanc-Benon, and D. Juvé, "Coherent function of a spherical acoustic wave after passing through a turbulent jet,' C. R. Acad. Sci., Ser. IIb: Mec., Phys., Chim., Astron. 326, 39 (1998).

${ }^{33}$ P. Petitjeans, J. E. Wesfreid, and J. C. Attiach, "Vortex stretching in a 
laminar boundary layer flow,' Exp. Fluids 22, 351 (1997).

${ }^{34}$ P. Petitjeans and J. E. Wesfreid, "Vortex stretching and filaments," Appl. Sci. Res. 57, 279 (1997)

${ }^{35}$ M. Fink, “Time-reserved acoustics," Phys. Today 50, 34 (1997).

${ }^{36} \mathrm{P}$. Roux, "Applications des miroirs acoustiques à retournement temporel à la focalisation dans un guide d'onde et à la caractérisation d'écoulements hydrodynamiques,"' Ph.D. thesis, University of Paris VI, 1996.

${ }^{37}$ C. Baudet, O. Michel, and W. J. Williams, "Detection of coherent vorticity structures using time-scale resolved acoustic spectroscopy," Physica D 128, 1 (1999). 